\title{
A scoring angioplasty balloon catheter for the management of complex tibial arterial lesions in critical lower limb ischaemia: A prospective study
}

\author{
Sherif Essam Tawfik, MD; Hesham Adel Alaa-Eldin MD
}

\author{
Department of General Surgery, Vascular Surgery Unit, Ain Shams University, \\ Cairo, Egypt.
}

\section{Correspondence:}

Sherif Essam Tawfik, Department of Surgery, Faculty of Medicine, Ain Shams University, Tel.: +20101601731; fax: +20226177003; E-mail address: stawfick@gmail.com

\begin{abstract}
Introduction: Complex tibial lesions represent a challenging problem in a large proportion of patients presenting with critical limb ischemia. In this particular subset of patients, heavily calcified and fibrocalcific lesions in the distal tibial tree, embodies an exigent problem for the success of the procedure.

Aim: To report our initial experience with the AngioSculpt ${ }^{T M}$ (ASC) scoring balloon catheter, which incorporates a laser-cut highly flexible three nitinol spiral struts which encircle a semicompliant conventional balloon catheter to create focal concentration of the dilating force leading to scoring (indenting) the surface of calcific plaques.

Methods: Patients scheduled for angioplasty of the tibial vessels for critical limb ischemia, were included in our study. Primary end points were limb salvage rate, clinical improvement, and primary patency. Secondary end points were, technical success, difficulties, and complications associated with the use of the scoring balloon.

Results: Forty Eight patients underwent peripheral angioplasty procedures in our institute, of which 11 patients with complex lesions fulfilled the inclusion criteria. Mean lesion length was $35 \pm 12.2 \mathrm{~mm}$, ostial lesion in $36.4 \%$ of patients. The Angiosculpt ${ }^{T M}$ balloon was used as sole therapy in all attempted tibial lesions. Limb salvage rate was $81.8 \%$. Primary patency rate of the treated vessels was $63.6 \%$ at 6 months.

Conclusion: The Angiosculpt ${ }^{T M}$ scoring balloon is a highly effective solution for challenging tibial complex lesions, and is associated with a very high technical success with low complication rate.
\end{abstract}

Keywords: Angioplasty, angioscore, angiosculpt, below the knee arteries, critical ischemia, infragenicular, scoring, tibial arteries.

\section{Introduction:}

Complex tibial lesions represent a challenging problem in a large proportion of patients presenting with critical limb ischemia. ${ }^{1}$ For most vascular surgeons tibial lesions represent the most challenging territory for angioplasty either because it is a technically demanding procedure or because of clinical considerations as usually most of these patients are suffering from critical lower limb ischemia (CLI) with a high potential for limb loss..$^{2,3}$

In this particular subset of patients with jeopardised limbs, tibial angioplasty has proven to be an efficient limb salvage procedure that can alleviate the need for a major surgical bypass procedure in such fragile group of patients. ${ }^{4-7}$ A substantial problem for the tibial angioplasty procedure is addressing the heavily calcified and fibrocalcific lesions in the distal tibial tree, which embodies an exigent problem for the success of the procedure. Thus there has been a great need for an angioplasty balloon that could address certain technical critical issues during the tibial angioplasty procedure, such as; low crossing profile balloon catheter for crossing tight lesions, high performance at 
low pressure with reduced local stress on the arterial wall to reduce neointimal hyperplasia, a uniform surface coverage with reduced balloon slippage to reduce the incidence of uncontrolled flow limiting dissections, as well as easy and quick balloon deflation and retrieval.

The Angiosculpt ${ }^{\mathrm{TM}}$ (ASC) scoring balloon catheter (AngioScore, Fremont, CA), incorporates a laser-cut highly flexible nitinol scoring element containing three spiral struts which encircle a semi-compliant conventional

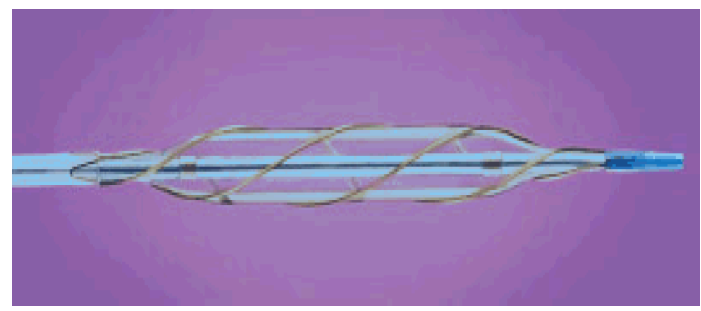

Figure (1): The Angiosculpt ${ }^{T M}$ scoring balloon.

We report our initial experience with the AngioSculpt ${ }^{\mathrm{TM}}$ (ASC) scoring balloon catheter in the treatment of critical limb ischemia in 11 patients with challenging tibial lesions at the Western Vascular Institute, Galway University Hospital, Ireland.

\section{Patients and methods:}

From our pool of patients scheduled for percutaneous transluminal angioplasty of the infrapopliteal vessels for critical limb ischemia, without associated proximal lesions amenable for angioplasty and were classified as Rutherford classification IV-VI, we included those who had challenging calcific lesions in our study.

The selection of challenging calcific lesions was dependant on a preoperative duplex scan conducted in a dedicated vascular lab, which was further confirmed by intraoperative preliminary angiography and identification of areas of calcification incurring radio opacity under fluoroscopy. Lesion calcification was graded according to the presence of radioopacities within the vascular wall at the site of the stenosis. The classification included none/ mild (no or minimal radio-opacities noted prior to contrast injection), moderate (multiple radio opacities noted prior to contrast injection), and severe (diffuse radio-opacities balloon catheter to create focal concentration of the dilating force along the edges of the scoring element Figure(1). This leads to more optimal luminal expansion compared to conventional balloon angioplasty, particularly in complex fibro-calcific lesions and may result in reduced barotrauma, less elastic recoil, and a lower incidence of uncontrolled dissections. The ASC is also designed to minimize device slippage and resultant vessel wall injury (i.e. "geographic miss") outside the intended treatment zone.

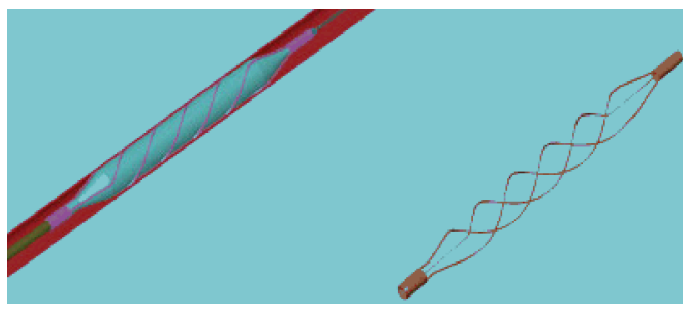

noted on both sides of the arterial wall).

Patient demographic data and pre-procedure baseline assessments of disease severity, including clinical classification of chronic limb ischemia based on the Rutherford Scale were recorded. ${ }^{8}$ Data regarding lesion characteristics; vessels treated, lesion location, and lesion angulation, were collected.

The ASC scoring balloon catheter was available in a rapid exchange delivery system with balloon diameter sizes 2.5, 3.0, and 3.5 $\mathrm{mm}$ and lengths of 15 , and $20 \mathrm{~mm}$. The size of the catheter was selected to approximate a $1: 1$ ratio to the reference vessel diameter to be treated. Under fluoroscopic control, a 0.014 guide wire was positioned beyond the target lesion and the ASC catheter advanced over the guide wire to the target lesion and was inflated to its nominal pressure for 2-3 minutes duration. Post dilatation angiography was done to evaluate the vessel diameter changes, recoil, dissection, or perforation

Primary end points were limb salvage rate, clinical improvement, and primary patency of the treated vessel segment by duplex scan over a period of six months follow up. Secondary end points were; (1) technical success judged by completion angio vessel diameter changes; (2) technical difficulties, as the need for stenting, recoil, or balloon slippage 
"geographical miss"; (3) technical complications as flow limiting dissection, thrombosis and perforation.

\section{Results:}

Between April 2005 and December 2005, 48 patients underwent peripheral angioplasty procedures in our institute, of which 11 patients were included in this study for fulfilling the inclusion criteria.
A total of 11 patients, with mean age of 75.4 years, five patients $(45.5 \%)$ were females. Patients were treated for 11 lesions in the tibial vessels territory. Seven out of the eleven patients were diabetics. Six patients were treated for minor tissue loss (Rutherford classification V) and five patients were treated for major tissue loss or gangrene (Rutherford classification VI). All patients were on aspirin, and statin prior to commencement of angioplasty procedures. Patients' demographic data and risk factors are shown in Table(1).

Table (1): Demographic data and risk factors.

\begin{tabular}{|l|l|}
\hline Number of patients & $\mathrm{n}=11$ \\
\hline Males : Females & $6: 5$ \\
\hline Age (years) & $75.4(59-85)$ \\
\hline Diabetes & $63.6 \%(\mathrm{n}=7)$ \\
\hline Hypertension & $36.4 \%(\mathrm{n}=4)$ \\
\hline Smoking & $54.5 \%(\mathrm{n}=6)$ \\
\hline Rutherford V & $54.5 \%(\mathrm{n}=6)$ \\
\hline Rutherford VI & $45.5 \%(\mathrm{n}=5)$ \\
\hline
\end{tabular}

The Angiosculpt $\mathrm{t}^{\mathrm{TM}}$ balloon was successfully deployed and used as sole therapy in all attempted tibial lesions Figure(2). Two out of the eleven patients had a previous bypass procedure; femoral to below knee popliteal bypass, and femoral to anterior-tibial bypass with a significant lesion in the tibial outflow vessel.
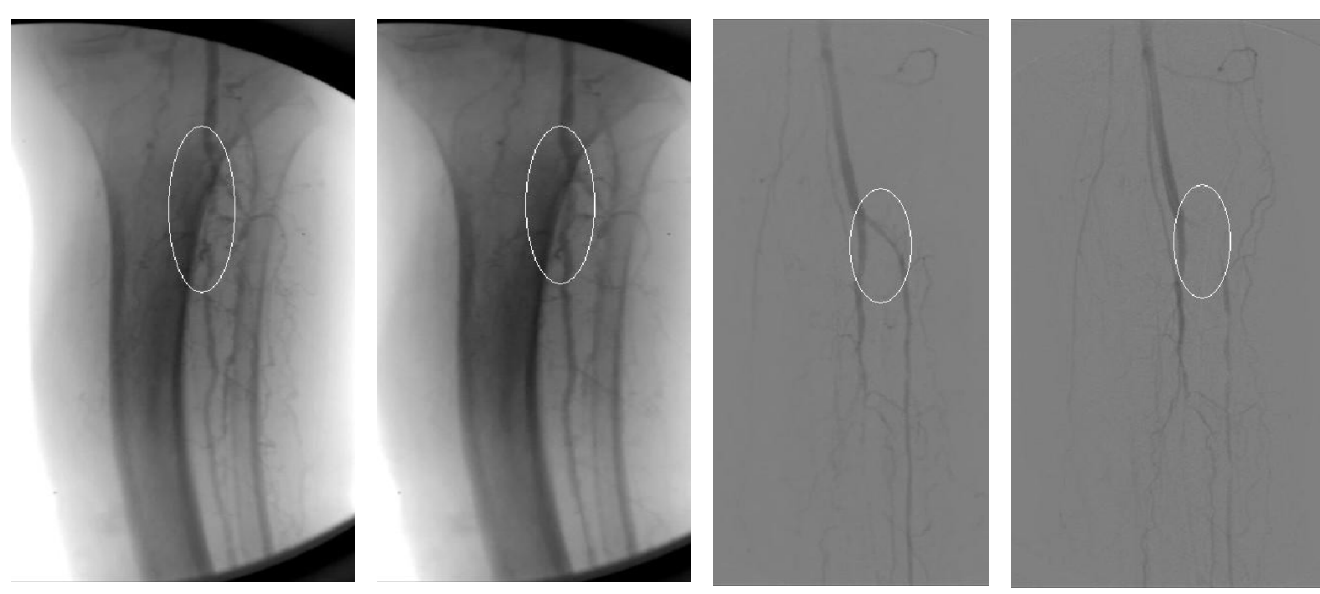

Figure (2): Pre and Post ASC angiography.

Lesion morphology was complex, including moderate/severe calcification in all eleven patients, lesion length was $35 \pm 12.2 \mathrm{~mm}$, ostial lesion in $4(36.4 \%)$ patients. Lesion and vessel characteristics are shown in Table(2). 
Table (2): Lesion and vessel characteristics.

\begin{tabular}{|l|l|}
\hline Treated lesions & $\mathrm{n}=11$ \\
\hline Mean lesion length $(\mathrm{mm})$ & $35 \pm 12.2$ \\
\hline Moderate/severe calcifications & $100 \%(\mathrm{n}=11)$ \\
\hline Occlusive lesions & $63.6 \%(\mathrm{n}=7)$ \\
\hline Stenotic lesions & $36.4 \%(\mathrm{n}=4)$ \\
\hline Ostial lesion & $36.4 \%(\mathrm{n}=4)$ \\
\hline Anterior tibial & $45.5 \%(\mathrm{n}=5)$ \\
\hline Peroneal/TPT & $36.4 \%(\mathrm{n}=4)$ \\
\hline Posterior tibial & $18.2 \%(\mathrm{n}=2)$ \\
\hline
\end{tabular}

TPT, tibio-peroneal trunk .

There were no technical difficulties encountered during the procedure, as all lesions responded to dilatation with no recoil or residual stenosis $>20 \%$ of the reference vessel diameter. There was no significant device slippage "geographical miss" during the ASC inflation.

Post-ASC technical complications in the form of dissections, was noted in only one (9\%) lesion and was minor that it didn't necessitate stenting. There were no perforations or instances of thrombosis encountered.

During follow up, 6 patients had minor amputations as part of planned primary procedure. Limb salvage rate was $81.8 \%$, with 2 major amputations during the follow up period for failure of symptomatic relief with intractable rest pain and spreading gangrene. Primary patency rate of the treated vessels was $63.6 \%(n=7)$ by duplex scan in our dedicated vascular lab. at 6 months follow up. The 30 day mortality was one patient with acute postoperative MI.

\section{Discussion:}

Patients presenting with infra-popliteal disease and CLI generally have severe multilevel and multivessel disease. It has been estimated that only $20-30 \%$ of such patients have an uncomplicated focal lesion with good distal run-off, and most are elderly with one or more comorbidities, which increases their surgical risk. ${ }^{9}$

As compared to conventional bypass surgery, PTA results in reduced rates of morbidity and mortality, shorter hospital stays and lower costs, does not preclude subsequent surgery and is rapidly becoming the treatment of first choice for infra-popliteal occlusive disease, particularly in patient populations at high risk for surgery. ${ }^{10}$

The known inadequacies associated with standard PTA balloons, include difficulty in treating heavily calcified lesions and diffuse small vessel disease. The technique also is associated with frequent balloon slippage resulting in "geographic miss" and tissue recoil. The results often are unpredictable and have a high rate $(\bullet 30 \%)$ of uncontrolled dissections. The ASC scoring balloon catheter may be helpful in addressing some of the limitations associated with conventional balloon angioplasty. The mechanism of action by which the ASC achieves luminal expansion include both circumferential scoring of the plaque as well as the effects of balloon dilatation. ${ }^{11,12}$ This may result in less baro-trauma, fewer and less severe dissections, leading to more predictable results particularly in fibro-calcific lesions. The relatively low dissection rate observed in this study compared to the historical experience with conventional balloons supports this contention. Scoring (indenting) the plaque may also reduce the likelihood of elastic recoil, particularly in ostial lesions and bifurcations. The absence of slippage and avoidance of ' "geographic miss' during device deployment which is generally accepted as favorable in the coronary circulation may also be beneficial in this setting. The favorable acute angiographic results associated with the use of the ASC in 
coronary studies may be particularly desirable in the treatment of infra-popliteal disease where avoidance of stenting particularly with balloon expandable compressible stents is preferred.

There have been several recent reports summarizing the short term clinical results with a number of other percutaneous technologies including cutting balloon, laserassisted angioplasty, and excisional atherectomy in patients presenting with similar peripheral arterial diseases. ${ }^{13-15}$

The advantages of the ASC balloon over the cutting balloon are, no longitudinal atherotomes thus can be inflated safely at higher pressures; the cutting balloon can be used for concentric lesions but cannot be used for eccentric lesions; the cutting balloon cannot be used for angulated lesions, for instance, ostial anterior tibial lesion.

The results of this initial clinical evaluation of the ASC scoring balloon catheter in the treatment of complex infra-popliteal disease and CLI demonstrate that the device is effective in a broad range of lesion morphologies and is associated with a very low complication rate. The device was demonstrated to be consistently deliverable throughout the affected arteries and resistant to slippage during deployment. This initial clinical experience with the ASC device in patients with infrapopliteal disease will need to be corroborated in larger multicenter studies that include adequate long term clinical follow-up.

\section{References:}

1- Diehm N, Shan A, Silvestro A, Do DD, Dick F, Schmidli J, et al: Association of cardiovascular risk factors with pattern of lower limb atherosclerosis in 2659 patients undergoing angioplasty. Eur J Vasc Endovasc Surg 2006; 31: 59-63.

2- Hafner J, Schaad I, Schneider E, Seifert B, Burg G, Cassina PC: Leg ulcers in peripheral arterial disease (arterial leg ulcers): Impaired wound healing above the threshold of chronic critical limb ischemia. J Am Acad Dermatol 2000; 43(6): 10011008.

3- Calle-Pascual AL, Duran A, Diaz A, Monux G, Serrano FJ, de la Torre NG, et al: Comparison of peripheral reconstruction in diabetic and non-diabetic patients: A prospective clinic based study. Diabetes Res Clin Pract 2001; 53(2): 129136.

4- Norgreen L, Hiatt WR, Dormandy JA, Nehler MR, Harris KA, Fowkes FGR (on behalf of the TASC II Working Group): Inter-society consensus for the management of peripheral arterial disease (TASC II). Eur J Vasc Endovasc Surg 2007; 33 (Suppl.1): S32-S55.

5- Haider SN, Kavanagh EG, Forlee M, Colgan MP, Madhavan P, Moore DJ, et al: Two-year outcome with preferential use of infra-inguinal angioplasty for critical ischemia. J Vasc Surg 2006; 43(3): 504512.

6- Dick F, Diehm N, Galimanis A, Husmann M, Schmidli J, Baumgartner I: Surgical or endovascular revascularization in patients with critical limb ischemia: Influence of diabetes mellitus on clinical outcome. J Vasc Surg 2007; 45: 751-761.

7- Lazaris AM, Tsiamis AC, Fishwick G, Bolia A, Bell PRF: Clinical outcome of primary infrainguinal subintimal angioplasty in diabetic patients with critical lower limb ischemia. J Endovasc Ther 2004; 11: 447-453.

8- Rutherford RB, Baker JD, Ernst C, et al: Recommended standards for reports dealing with lower extremity ischemia: Revised version. J Vasc Surg 1997; 26: 517-538.

9- Heuser R, Henry M: Textbook of peripheral vascular interventions. London: Taylor \& Francis Group; 2004; p.555.

10-BASIL trial participants: Bypass versus angioplasty in severe ischaemia of the leg (BASIL): Multicentre, randomized controlled trial. Lancet 2005; 366: 1925 1934.

11-Gershony G, Virmani R, Lotan C, et al: A novel angioplasty catheter for the treatment of complex CAD: AngioSculpt. Am J Cardiol 2003; 92: 166L.

12-Ferreira EJ, Vaz VD, Abizaid A, et al: A novel scoring catheter balloon (AngioSculpt) for the treatment of complex coronary lesions: An intravascular ultrasound study. Am J Cardiol 2004; 94: 195E-196E. 
13-Ansel GM, Sample NS, Botti CF, et al: Cutting balloon angioplasty of the popliteal and infrapopliteal vessels for symptomatic limb ischemia. Catheter Cardiovasc Interv 2004; 61: 1-4.

14-Laird JR, Zeller T, Gray BH, et al: Limb salvage following laser-assisted angioplasty for critical limb ischemia: Results of the LACI multicenter trial. $J$ Endovasc Ther 2006; 13:1-11.
15-Kandzari DE, Kiesz RS, Allie D, et al: Procedural and clinical outcomes with catheter-based plaque excision in critical limb ischemia. J Endovasc Ther 2006; 13: 12-22. 